Rapid Reviews COVID-19

\title{
Reviews of "Sensitivity of SARS-CoV-2 antigen- detecting rapid tests for Omicron variant"
}

Julian Tang ${ }^{1}$, Monika Klimek-Tulwin ${ }^{2}$

${ }^{1}$ University of Leicester, ${ }^{2}$ Medical University of Lublin

Published on: Mar 03, 2022

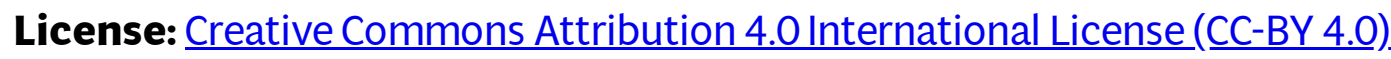


To read the original manuscript, click the link above.

Summary of Reviews: This preprint evaluates the sensitivity of various antigen-based COVID-19 diagnostic kits and found variable sensitivity for patients infected with the Omicron variant-of-concern. Reviewers deemed this study potentially informative, pointing out limitations with frozen samples.

\section{Reviewer 1 (Julian Tang)}

\section{Reviewer 2 (Monika Klimek-Tulwin)}

\section{RR:C19 Strength of Evidence Scale Key}

$$
\begin{aligned}
& \text { प }
\end{aligned}
$$

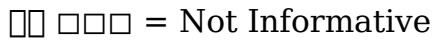

$$
\begin{aligned}
& \text { प्रा पि = Potentially Informative }
\end{aligned}
$$

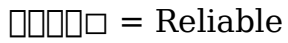

$$
\begin{aligned}
& \text { प्राप्र = Strong }
\end{aligned}
$$

To read the reviews, click the links below. 